\title{
Melioidosis: Evolving Concepts in Epidemiology, Pathogenesis, and Treatment
}

\author{
Bart J. Currie, FRACP ${ }^{1}$ \\ ${ }^{1}$ Global and Tropical Health Division, Menzies School of Health \\ Research and Infectious Diseases Department, Royal Darwin \\ Hospital, Darwin, Australia \\ Semin Respir Crit Care Med 2015;36:111-125.
}

\begin{abstract}
Address for correspondence Bart Currie, FRACP, Menzies School of Health Research, PO Box 41096, Casuarina, NT 0811

(e-mail: bart.currie@menzies.edu.au).
\end{abstract}

\begin{abstract}
Infection with Burkholderia pseudomallei can result in asymptomatic seroconversion, a single skin lesion that may or may not heal spontaneously, a pneumonia which can be subacute or chronic and mimic tuberculosis or rapidly progressive resulting in fatal overwhelming sepsis. Latency with subsequent activation of disease is well recognized, but very uncommon. Melioidosis also has a myriad of other clinical presentations and diagnosis is often delayed because of this and because of difficulties with laboratory diagnosis and lack of recognition outside melioidosis-endemic regions. The perception of $B$. pseudomallei as a top tier biothreat agent has driven large funding for research, yet resources for diagnosis and therapy of melioidosis in many endemic locations remain extremely limited, with mortality as high as $50 \%$ in comparison to around $10 \%$ in regions where state-of-the-art intensive care therapy for sepsis is available. Fatal melioidosis is extremely unlikely from natural infection in a healthy person, provided the diagnosis is made early, ceftazidime or meropenem is commenced and intensive care therapy is available. While biothreat research is directed toward potential aerosol exposure to $B$. pseudomallei, the overall proportion of melioidosis cases resulting from inhalation rather than from percutaneous inoculation remains entirely uncertain, although the epidemio-

Keywords

- melioidosis

- Burkholderia pseudomallei

- sepsis

- therapy

- antimicrobial resistance

- biothreat logy supports a shift to inhalation during severe weather events such as cyclones and typhoons. What makes $B$. pseudomallei such a dangerous organism for patients with diabetes and other selective risk factors remains unclear, but microbial genome-wide association studies linking clinical aspects of melioidosis cases to nonubiquitous or polymorphic $B$. pseudomallei genes or genomic islands are beginning to uncover specific virulence signatures. Finally, what also remains uncertain is the global phylogeography of $B$. pseudomallei and whether melioidosis is spreading beyond historical locations or is just being unmasked in Africa and the Americas by better recognition and increased surveillance.
\end{abstract}

\section{Melioidosis, the Burkholderia genus and Burkholderia pseudomallei}

Melioidosis is an infectious disease of humans and animals resulting from infection with the gram-negative soil and water bacterium, Burkholderia pseudomallei. ${ }^{1-4}$ The genus Burkholderia is composed of a rapidly increasing number of recognized species, with many having specific environmental niches and often complex interactions with other microorganisms and plants. ${ }^{5}$ Various Burkholderia species have shown potential for biocontrol, bioremediation, and plant growth promotion, while others are recognized plant pathogens. ${ }^{6}$ Most recently, an isolate recovered from the tropical savannah environment of the Northern Territory of Australia
Issue Theme Antimicrobial Resistance: Management of Superbugs; Guest Editor, David L. Paterson, MBBS, PhD, FRACP, FRCPA
Copyright (c) 2015 by Thieme Medical Publishers, Inc., 333 Seventh Avenue, New York, NY 10001, USA. Tel: +1(212) 584-4662.
DOI http://dx.doi.org/ 10.1055/s-0034-1398389. ISSN $1069-3424$. 
(MSMB43, with proposed new species name Burkholderia humptydooensis) has been shown to produce a remarkable array of novel natural compounds, including some exhibiting potentially potent antitumor activity through inhibition of pre-mRNA splicing. ${ }^{7,8}$

There is also an enormous diversity of antimicrobial susceptibility profiles and antimicrobial resistance mechanisms among the species in the Burkholderia genus. Within the genus the notable pathogens for humans or animals are limited to B. pseudomallei, Burkholderia mallei, the agent of equine ganders, and various species from the Burkholderia cepacia complex which are nosocomial pathogens for immunocompromised individuals and can also have devastating consequences once they colonize the airways of persons with cystic fibrosis. ${ }^{9}$

\section{Historical Aspects}

In 1912, Whitmore and Krishnaswami described a newly recognized septicemic disease in morphine addicts in Rangoon, Burma. ${ }^{10}$ Fatal cases had extensive caseous consolidation of the lung and abscesses in liver, spleen, kidney, and subcutaneous tissues. They isolated a bacillus that was similar to that causing glanders except that it was motile. Whitmore noted the clinical similarity to glanders, and Stanton and Fletcher subsequently proposed the name melioidosis, derived from the Greek melis (distemper of asses). ${ }^{11}$

Of note glanders was described by Hippocrates and had long been recognized as an occupational risk for horse handlers and equine butchers, and in more recent times veterinarians and laboratory workers. Despite glanders being recognized for centuries before melioidosis was first described, multilocus sequence typing (MLST) ${ }^{12}$ and whole genome sequencing ${ }^{13,14}$ have shown that $B$. mallei is a clone of $B$. pseudomallei, with a considerably smaller genome reflecting the genome reduction that occurred in the evolution from the environmental $B$. pseudomallei to the mammalian-adapted pathogen $B$. mallei that is no longer able to survive in the environment outside its equine host. The hostadaptation of $B$. mallei likely explains why it appears to be more easily transmitted nosocomially than $B$. pseudomallei, such as occurs with laboratory-acquired infections. ${ }^{15}$ It also likely contributes to why $B$. mallei may be more able to cause severe disease in healthy human hosts than B. pseudomallei; in case series from locations with resources for rapid laboratory diagnosis and state-of-the-art therapy for severe sepsis, fatal melioidosis is almost always restricted to those with identified risk factors. ${ }^{16}$

Glanders together with anthrax was implicated in the first modern era biological warfare, with attempts between 1915 and 1918 to infect horses in the United States, Romania, Spain, Norway, and Argentina. ${ }^{17}$ Since, 2001 there has been an exponential increase in research on $B$. pseudomallei and melioidosis, driven in a large part because of the perceived potential use of $B$. pseudomallei as a biological weapon. In October 2012, B. pseudomallei was upgraded to a Tier 1 Select Agent by the Centers for Disease Control and Prevention. ${ }^{18}$ Concerns of a deliberate release of $B$. pseudomallei are based around the high mortality rate in published studies, the ability for $B$. pseudomallei to be easily aerosolized in the laboratory setting, intrinsic resistance to standard antimicrobial agents, the current lack of a vaccine and the diverse range of disease presentations that confound and delay diagnosis, particularly in nonendemic regions.

\section{Global Distribution of Melioidosis: Recent Expansion or Just Unmasking of $B$. pseudomallei?}

Melioidosis occurs predominantly in Southeast Asia, northern Australia, South Asia and China and Taiwan. ${ }^{3,19}$ Despite being first described in 1912 in Burma, then Malaysia and Singapore from 1913, Vietnam from 1925 and Indonesia from 1929, melioidosis was not documented in Thailand until 1955 and in Australia until 1949, despite these two countries having the endemic regions with the highest documented incidence rates. Thailand has by far the largest number of melioidosis cases with an estimated 2,000 to 3,000 cases of melioidosis each year, ${ }^{20}$ with most cases documented from the northeastern provinces. ${ }^{21,22}$ The tropical "Top End" of the Northern Territory of Australia has had a surge of cases since two particularly heavy consecutive monsoonal wet seasons beginning late 2009, with an incidence rate of 50.2 cases per 100,000 in the Top End population overall in 2009 to $2010 .{ }^{23}$ Melioidosis is now well recognized in and case series documented from Singapore, Malaysia, Brunei, Laos, Cambodia, Vietnam, China (Hainan province and Hong Kong), Taiwan, India, and Sri Lanka.

Maps of the global distribution of melioidosis have been published and updated. ${ }^{1,24}$ Small case numbers have been documented from the Philippines, Papua New Guinea, and the Pacific Ocean islands of New Caledonia. Cases of melioidosis are increasingly being documented from elsewhere outside the classic endemic region, with sporadic human or animal cases or environmental isolates of B. pseudomallei from the Middle East, Africa, the Indian Ocean islands of Mauritius, La Reunion and Madagascar, the Caribbean, and Central and South America. ${ }^{25-28}$

Despite recent cases from Brazil, Puerto Rico, and various African countries, the true extent and magnitude of the presence of B. pseudomallei in the Caribbean, Central, and South America and Africa remains entirely unknown. Furthermore, it remains to be elucidated as to whether the recent expansions of known endemic locations reflects just unmasking of longer standing presence of $B$. pseudomallei in the local environment or if there is substantial dissemination occurring as a result of increasing human and/or animal and plant and soil movements globally. Global warming may well result in expansion of the endemic boundaries of melioidosis. Melioidosis endemic locations may vary in their specific ecological niches for B. pseudomallei. For example, B. pseudomallei has been shown to colonize and thrives in the rhizosphere and aerial parts of native and imported grasses in northern Australia, raising implications for global epidemiology and potential dispersal. ${ }^{29}$

The origins and global dispersal of B. pseudomallei also require further elucidation. It is likely that $B$. pseudomallei evolved from an ancestral environmental Burkholderia species and survived and proliferated in an as yet undetermined 
environmental niche of its own, possibly providing a biodefense role supporting cohabiting plant species. Phylogeographic reconstruction of $B$. pseudomallei genomes based on combined analysis of single nucleotide polymorphisms from whole genome sequencing and analysis of sequence data from the seven MLST loci supported an Australian origin of B. pseudomallei, with possibly a single introduction event into Southeast Asia. ${ }^{30}$ This may have occurred during the last ice age when low sea levels resulted in land bridges between what are now islands in the Malay Archipelago. However, the study focused on strains from Australia and Southeast Asia and included few strains from the rest of the world. A recent analysis of strains from the Caribbean, Central and South America showed a consistent presence of the to-date otherwise uncommon type $G$ pattern in the 16S-23S internal transcribed spacer of $B$. pseudomallei. ${ }^{31}$ This type $G$ pattern has also been found in strains from Africa and it was proposed that these findings were consistent with the hypothesis that the type $G$ isolates may reflect a bottleneck that occurred during the dispersal of B. pseudomallei from Southeast Asia to the rest of the world. However, MLST analysis of strains with the type $G$ pattern shows considerable diversity and most recently two confirmed autochthonous cases of human melioidosis in Madagascar and a third case in a traveler infected in Madagascar each had novel MLST sequence types. ${ }^{27}$ Therefore, more ancient origins of $B$. pseudomallei could conceivably also explain the presence of $B$. pseudomallei of novel and diverse genotypes in Africa and in the Americas. Whole genome sequencing of multiple isolates from diverse locations is currently underway and should help resolve the origins and global dispersal patterns of $B$. pseudomallei.

With improved clinical surveillance and laboratory diagnosis an increasing number of cases of melioidosis is being reported in people infected in an endemic region who return or travel to Europe, the United States and elsewhere. ${ }^{32}$ Tourists with diabetes and cystic fibrosis are especially at risk if exposed to wet season soil and surface water and monsoonal storms where aerosolization of $B$. pseudomalle $i$ is suspected to occur. ${ }^{33}$

Three recent cases $(2010,2011,2013)$ and two historical cases $(1950,1971)$ of melioidosis have occurred in the United States in people with no history of overseas travel to endemic regions. ${ }^{34-36}$ Because of the potential biothreat scenario the recent cases have been extensively investigated to ascertain a source of infection. Despite comprehensive personal and family history investigations and extensive sampling for $B$. pseudomallei in the domestic environments using culture and real-time polymerase chain reaction (PCR), including sampling of pets, insects, soil, and houseplants, the source of the infection was unable to be identified in any of the cases. The MLST sequence type of $B$. pseudomallei from each of the three cases was different but on interrogation of the global MLST database (http://bpseudomallei.mlst.net/), each was linked to a Southeast Asian origin. Therefore, in each case it is likely that exposure resulted from a source brought from Southeast Asia to the United States, with importation of exotic reptiles, plants or plant products or contaminated medicinal products all possible. With globalization of the medicinal products market and recognition that contamination of antiseptics and intravenous solutions with $B$. pseudomallei has resulted in past clusters of melioidosis, vigilance is required to diagnose such scenarios quickly. Diagnosis and therefore appropriate therapy in the most recent and eventually fatal case in the United States was delayed by over a month because the initial $B$. pseudomallei isolated from blood was misidentified. $^{34}$

\section{Epidemiology: How Important is Inhalation and How Common is Latency?}

Transmission of $B$. pseudomallei occurs primarily through percutaneous inoculation and inhalation/aspiration. Ingestion is a common route of infection in grazing animals and a recent matched case-control study from Thailand suggested that for humans, ingestion of $B$. pseudomallei from unchlorinated domestic water supplies and other water sources such as rivers may be more common than previously thought. ${ }^{37}$ This is supported by two outbreaks of melioidosis in Australia that were linked to contamination of potable water with $B$. pseudomallei, with the implicated water supplies being unchlorinated or with chlorine levels below standard. ${ }^{38,39}$ Transmission to infants through ingestion of breast milk from mothers with melioidosis mastitis has been described. ${ }^{40}$ Vertical and sexual transmission, ${ }^{41}$ zoonotic transmission from animals with melioidosis ${ }^{42}$ and nosocomial infection and transmission to laboratory staff are all very uncommon but documented.

During severe weather events such as tropical monsoonal storms, cyclones (southern hemisphere), hurricanes (northern hemisphere), and typhoons (e.g., Hong Kong, Taiwan, Philippines), there may be a shift from inoculation to inhalation as the predominant route of $B$. pseudomallei transmission. ${ }^{4-47}$ Rainfall in the 2 weeks before the onset of symptoms is an independent risk factor for a pneumonic presentation, septic shock, and death, supporting a shift to inhalation. ${ }^{45}$ Nevertheless, melioidosis pneumonia is also well recognized to occur after documented inoculating skin injuries such as soil-contaminated burns, with pneumonia considered to result from hematogenous spread of $B$. pseudomallei from the inoculation site to the lungs. ${ }^{48}$ Melioidosis pneumonia was documented following the devastating December 2004 Indian Ocean tsunami and these "tsunami lung" cases were mostly associated with aspiration events, though inoculation of the organism in the setting of lacerations also may have occurred. ${ }^{49-51}$

The potential for inhalation of $B$. pseudomallei was first raised in the descriptions of melioidosis pneumonia in soldiers exposed to dusts raised by helicopter rotor blades in Vietnam ${ }^{52}$ and helicopter-associated inhalation was implicated in a recent case in a tourist returned from Singapore. ${ }^{53}$ Inhalational melioidosis is also supported by the increasing recognition from computed tomography (CT) scanning that mediastinal lymph nodes are not uncommonly seen in melioidosis pneumonia. Animal studies also support the potential importance of aerosol inhalation, with high lethality seen. ${ }^{54-57}$ Recently, an evidence-based clinical definition of inhalational melioidosis has been described to provide a more 
focused definition for biothreat-related research and assist those organizations who develop guidelines on emergency response after a deliberate release of biothreat agents. ${ }^{58}$ Nevertheless, the overall proportion of melioidosis cases resulting from inhalation rather than percutaneous inoculation remains entirely uncertain and may well vary substantially between locations and seasons depending on occupational and recreational profiles and weather patterns.

Exposure to $B$. pseudomallei most commonly results in subclinical disease with or without seroconversion. In northeast Thailand modeling of serology data suggested that only $1: 4,600$ antibody-producing exposures result in clinical infection in that region. ${ }^{59}$ However, the background seropositivity rate in parts of northeast Thailand has been documented to exceed $50 \%$, with most seroconversion occurring between 6 months and 4 years of age. ${ }^{60}$ This seropositivity rate is far greater than that seen in tropical Australia, where the incidence rates of confirmed melioidosis are similar to those seen in northeast Thailand, yet overall seropositivity rates are under $5 \%{ }^{61,62}$ Furthermore, the absolute case numbers from the Darwin prospective melioidosis study from the tropical "Top End" of the Northern Territory of Australia show that in each of two recent years 1 in every 2,000 people living in the Top End had cultureconfirmed melioidosis despite the low seropositivity rates seen. ${ }^{23}$ The massive disparity between the Thai model of infection/seropositivity/clinical disease and the data from Australia reflects a large gap in our understanding of the epidemiology of infection with and disease from B. pseudomallei. This is mirrored in the uncertainty of how many asymptomatic people with positive serology, presumably reflecting infection with $B$. pseudomallei at some time point, have not cleared their infection and have bacteria still present in undetermined latent foci. Furthermore, of those with latent infection, how many will subsequently have activation of infection resulting in clinical disease (i.e., melioidosis)? The latter concern was coined the "Vietnamese time bomb," with estimates from serology studies of $\sim 225,000$ potential cases in United States military personnel returning from the Vietnam war. ${ }^{63}$ However, while occasional cases of activation of $B$. pseudomallei from latency still occur in Vietnam veterans, it is rare in comparison to the vast numbers exposed.

Over the 25 years of the Darwin prospective melioidosis study it is estimated that fewer than $4 \%$ of the cases were potentially activation from latency, with the vast majority of cases being recent infection progressing to clinical disease. $^{16,64}$ The predominance of recent infection is supported by the strongly seasonal nature of melioidosis, with $75 \%$ of the cases in northeast Thailand ${ }^{65}$ and $81 \%$ of the cases in the Northern Territory of Australia ${ }^{16}$ occurring during the monsoonal wet season. Nevertheless, vigilance for activation from latency remains important, with the longest well documented latent periods between exposure in an endemic region and the development of melioidosis in a nonendemic region being 62 years in the United States (a returned WWII prisoner of war in Burma-Thailand-Malaysia) ${ }^{66}$ and 24 years in southern Australia. ${ }^{67}$

\section{Risk Factors for Melioidosis and Pathogenesis: Healthy People Shouldn't Die from Melioidosis?}

The clinical presentations, severity of disease and outcomes in melioidosis are all influenced by bacterial load on exposure (infecting dose), route of infection (see above for inhalation vs. percutaneous inoculation), virulence of the infecting strain of $B$. pseudomallei and most importantly the human host risk factors. With rapid diagnosis, appropriate antibiotics, and state-of-the-art management of sepsis, death from melioidosis in those without identified risk factors for melioidosis is actually extremely uncommon. In the Darwin prospective melioidosis study 113 of the 115 fatalities directly attributable to melioidosis over the 25 years to date have occurred in patients with one or more known risk factors for melioidosis (updated from Currie et al16). It has also been noted that despite the large bacterial load in severely ill patients with septicemic pulmonary melioidosis, person-toperson transmission is extremely unusual, further supporting the primary importance of host risk factors for development of melioidosis.

Up to $80 \%$ of patients overall have one or more risk factor for melioidosis, although in children, who account for 5 to $15 \%$ of all the cases, risk factors are far less common, being present in only $16 \%$ of Australian children with melioidosis. ${ }^{68}$ Risk factors include diabetes (23-60\% of patients), hazardous alcohol use (12-39\%), chronic lung disease (12-27\%), chronic renal disease (10-27\%), thalassemia (7\%), glucocorticoid and other immunosuppressive therapy $(<5 \%)$, and cancer $(<5 \%)^{16,22,69-71}$ In Thailand, the adjusted odds ratios for diabetes and renal disease (chronic renal impairment or renal or ureteric calculi) in cases of melioidosis versus controls were 12.9 (95\% confidence interval [CI], 5.1-37.2) and 2.9 (95\% CI, 1.7-5.0), respectively. ${ }^{65}$

The predisposition to melioidosis in those with diabetes, hazardous alcohol use, or chronic renal disease, likely reflects impairment of their innate immune function, especially neutrophil and other phagocytic cell functions, such as mobilization, delivery, adherence, and ingestion and killing. ${ }^{71,72}$ The immunopathogenesis of melioidosis has been extensively studied, with tightly regulated Toll-like receptor responses from host cells seen and other pathogen recognition receptor/response mechanisms described. ${ }^{1,73-76}$ There are important host protective mechanisms against $B$. pseudomallei in cytokine responses (especially adequate production of interferon- $\gamma$ ) as well as potentially detrimental ones, with the timing of cytokine release and the balance between pro- and anti-inflammatory responses likely to determine the severity of disease and outcome of infection.

In Thailand there was an association of severe melioidosis with tumor necrosis factor (TNF)- $\alpha$ gene allele $2,{ }^{77}$ which is linked to higher constitutive and inducible production of TNF- $\alpha$. Recently, a common genetic polymorphism in the NOD2 region, rs7194886, was found to be associated with melioidosis. ${ }^{76}$ Although functional genetic variations in TLR1 have been associated with death from sepsis in white Americans, TLR4 and TLR5 genetic variations but not TLR1 genetic variations have been linked to outcomes for melioidosis in the Thai population. ${ }^{75,78-81}$ Nevertheless, the rarity of fatal 
melioidosis in patients without risk factors in the Darwin prospective melioidosis study suggests that these and other yet to be determined variable genetic polymorphisms in human innate immune responses are likely to be considerably less important overall in determining outcomes from melioidosis than the presence of one or more of the recognized risk factors for melioidosis. ${ }^{16}$

Although a vigorous cell-mediated immune response may protect against disease progression, ${ }^{82,83}$ infection with human immunodeficiency virus does not appear to be a risk factor for developing melioidosis or for more severe disease or a fatal outcome. ${ }^{84}$ Furthermore, there is no definitive evidence for the development of functional immunity from melioidosis after infection with $B$. pseudomallei and reinfection can occur with a different strain of $B$. pseudomallei after successful treatment of melioidosis. ${ }^{70,85}$

B. pseudomallei has two chromosomes, with a large accessory genome that includes multiple genomic islands that are variably present in different strains and have a great propensity for horizontal gene transfer. ${ }^{13,86,87}$ However, the specific virulence mechanisms that make $B$. pseudomallei such a dangerous pathogen for hosts with risk factors are poorly elucidated. B. pseudomallei is a facultative intracellular pathogen that invades and replicates inside polymorphonuclear leukocytes, macrophages, and some epithelial cell lines. $B$. pseudomallei capsular polysaccharide is important in intracellular survival of the bacteria ${ }^{88}$ and lipopolysaccharide confers resistance to human serum. ${ }^{89}$ Type III and Type VI secretion systems in B. pseudomallei are also important in cell invasion and intracellular survival. ${ }^{90,91}$ Quorum sensing has diverse potential roles in the virulence of B. pseudomallei, including cell invasion, cytotoxicity, and antimicrobial resistance. ${ }^{92}$ Other proposed virulence factors include a siderophore, secreted proteins such as proteases, lipases and hemolysin, flagella, type IV pili, and other adhesins. Burkholderia lethal factor-1 was recently described and is similar to Escherichia coli cytotoxic necrotizing factor-1, with interference with initiation of translation resulting in alteration of the actin cytoskeleton and ultimately cell death. ${ }^{93}$ An additional survival factor for B. pseudomallei is the ability for phenotypic switching with a change in colony morphology, resulting in changes in the expression of putative virulence factors such as biofilm and flagella. ${ }^{94,95}$

Although laboratory rodent studies of isolates of B. pseudomallei from animals, humans, and the environment have shown that virulence can differ between $B$. pseudomallei strains, ${ }^{96}$ the importance of this variation in virulence in determining clinical aspects of melioidosis in humans remains uncertain. In both animal and human clusters confirmed by genotyping to be from a clonal strain of $B$. pseudomallei, the same outbreak strain can cause very different clinical presentations and outcomes, with host risk factors being most important in determining the severity of disease. ${ }^{39}$

The previously described virulence factors are ubiquitous amongst B. pseudomallei strains and any molecular basis for differential pathogenesis and virulence between strains had remained elusive until a recent study linking clinical data from 556 melioidosis patients to B. pseudomallei genes which are either variably present or polymorphic. ${ }^{97}$ That study identified a $B$. mallei-like actin polymerization bimABm gene that is strongly associated with neurological disease and also a filamentous hemagglutinin gene, fhaB3, which is associated with positive blood cultures but is negatively correlated with localized skin lesions without sepsis. Ongoing microbial genome-wide association studies linking clinical aspects of melioidosis cases to nonubiquitous or polymorphic B. pseudomallei genes or genomic islands are likely to uncover additional virulence signatures.

\section{Clinical Manifestations: Enormous Diversity but Pneumonia Predominates}

As noted previously, infection with B. pseudomallei usually results in subclinical disease. In those who develop melioidosis, disease presentation, severity, and outcome are all substantially influenced by the presence or absence of risk factors and route of infection, with infecting bacterial load and in select cases the presence or absence of specific nonubiquitous $B$. pseudomallei virulence genes. The clinical spectrum of disease ranges from localized cutaneous infection at an inoculation site with no systemic manifestations to overwhelming sepsis and death. The incubation period ranges from 1 to 21 days (mean 9 days) for acute presentations, ${ }^{48}$ which represent $85 \%$ of all cases in the large prospective study from northern Australia. ${ }^{16}$ Onset of melioidosis within 24 hours has been seen in presumed aspiration after neardrowning and, in some cases, after severe weather events. ${ }^{98,99}$ Bacteremia on admission occurs in 40 to $60 \%$ of the cases, septic shock in around $20 \%$ and pneumonia is the presenting illness in over half of all cases. Mortality rates overall are currently between $10 \%$ in Australia and $40 \%$ in Thailand, ${ }^{21}$ with still higher rates in resource-poor areas where access to diagnosis and therapy are limited. Before ceftazidime and then goal-directed therapy for severe sepsis were implemented for melioidosis at Royal Darwin Hospital in the 1990s, melioidosis with septic shock requiring ventilation in the intensive care unit was universally fatal.

Around $11 \%$ of the cases present with chronic melioidosis, defined as illness with symptoms for longer than 2 months' duration on presentation. ${ }^{16}$ The two major presentations of chronic melioidosis are pneumonia or nonhealing skin ulcers/ abscesses (usually but not always a single lesion) that have failed to respond to antistaphylococcal therapy. The former patients often have features mimicking tuberculosis, with weight loss, fevers, night sweats, productive cough sometimes with hemoptysis and classically upper lobe infiltrates, with or without cavitation on chest X-ray or CT scan. Disease can be remitting and relapsing over months or even years and is not uncommonly misdiagnosed as tuberculosis. Acute deterioration with septicemia can occur in these patients but overall mortality in chronic melioidosis is low. As discussed earlier the final $4 \%$ of the cases represent activation from latent infection and these patients can present as localized or disseminated disease.

As noted, pneumonia is the most common clinical presentation of patients with melioidosis in all studies, accounting 
for over half of the cases and with both acute and chronic manifestations possible. ${ }^{33,100}$ Secondary pneumonia after another primary presentation occurs in around $10 \%$ of the cases. Acute melioidosis pneumonia has a spectrum from overwhelming septic shock with mortality up to $90 \%$, to mild undifferentiated pneumonia, which can be acute or subacute in nature, with little mortality. Septicemic patients present acutely unwell with prostration, high fevers, and often little initial cough or pleuritic pain. Chest X-ray may initially have limited infiltrates but can then rapidly progress to extensive unilateral consolidation or diffuse nodular infiltrates throughout both lungs. These infiltrates then coalesce and cavitate, consistent with the caseous necrosis and multiple metastatic abscess formation seen at autopsy. Nonsepticemic patients with pneumonia have a more predominant cough, with productive sputum and dyspnea, and their chest X-rays show discrete but progressive consolidation in one or more lobes. In endemic regions and in travelers returned from endemic regions, acute pneumonia with upper lobe consolidation warrants consideration of melioidosis, although lower lobe infiltrates are also common.

In the Darwin prospective study $13 \%$ of patients overall ( $28 \%$ of patients without bacteremia) present with skin ulcers or abscesses which are usually but not always single lesions at an inoculation site that are indistinguishable from staphylococcal or other bacterial or fungal skin lesions and have often failed to respond to oral antibiotics administered before admission. ${ }^{101}$ Cellulitis is, however, very uncommon. Primary cutaneous melioidosis is the most common presentation for children with melioidosis in northern Australia, with $60 \%$ of children presenting with skin lesion(s), while in contrast to adults only $20 \%$ of children present with pneumonia. ${ }^{68}$ Of note, in Thailand and Cambodia the commonest presentation for children with melioidosis is suppurative parotitis, which has rarely been seen in Australia. ${ }^{102,103}$ This regionally specific occurrence of parotitis may reflect ingestion or aspiration of B. pseudomalleicontaminated water from unchlorinated domestic water supplies and other water sources. ${ }^{37}$

In Australia genitourinary melioidosis appears much more common than in Southeast Asia and accounts for $14 \%$ of all presentations. ${ }^{16}$ It occurs predominantly in males with development of prostatic abscesses. ${ }^{104}$ Presentation is with fever in association with suprapubic pain, dysuria, difficulty passing urine, or acute urinary retention requiring catheterization. Diarrhea frequently accompanies these symptoms. A tender, boggy prostate may be found on rectal examination. Prostatic abscesses can also be present on admission or develop in a patient who has presented with another primary diagnosis, usually pneumonia. Around $18 \%$ of all the males in Australia with melioidosis are found to have prostatic abscesses.

Presentation with bacteremia without a clinically evident focus is being increasingly seen in Australia and currently accounts for 10 to $15 \%$ of the cases. It is particularly common in patients on immunosuppressive therapy for hematological and solid organ malignancies and in those on hemodialysis for chronic renal failure, potentially reflecting rapid progression to bacteremia after infection occurs in a host with poor innate immunity. Presentations with bacteremia without a clinically evident focus are likely to increase as more people in endemic regions have access to more aggressive cancer chemotherapy regimens and to dialysis. Clinical vigilance and in some cases chemoprophylaxis against melioidosis with trimethoprimsulfamethoxazole are being incorporated into management regimens for these patients. ${ }^{105}$

An uncommon but important presentation of melioidosis in Australia is encephalomyelitis, accounting for approximately $4 \%$ of melioidosis cases in northern Australia. ${ }^{106-108}$ This clinical syndrome appears to be far less common in Southeast Asia, where brain involvement in melioidosis is usually cerebral abscesses secondary to bacteremic spread, which is also seen in Australia. ${ }^{109}$ The clinical presentation of melioidosis encephalomyelitis usually reflects primarily brainstem infection and almost all have a normal or nearnormal initial state of consciousness. CT scan is often normal but increased T2 signal is invariably seen on magnetic resonance imaging, often extending beyond those areas of brainstem and spinal cord suggested by the clinical findings. Prominent features include unilateral upper motor neuron limb weakness, cerebellar signs, cranial nerve palsies (particularly VI, VII nerve palsies, and bulbar palsy). Some cases present with flaccid paraparesis alone. Despite most patients with melioidosis encephalomyelitis having no identified risk factors for melioidosis, severe residual neurological deficits can occur, including in children. ${ }^{68}$ Studies in mice have supported the hypothesis that melioidosis encephalomyelitis may result from $B$. pseudomallei directly invading the brain via movement along olfactory and/or trigeminal nerve root pathways following colonization of the nasal mucosa. ${ }^{110,111}$ The possibility has also been raised of direct brainstem or spinal cord infection via nerve root translocation of bacteria secondary to skin inoculation with $B$. pseudomallei on the face/scalp or limbs, respectively. ${ }^{68}$ As already noted, recent analysis of $B$. pseudomallei isolates has shown a correlation between presentation with encephalomyelitis and the presence of the $B$. mallei-like actin polymerization bimABm gene; a gene that has rarely been found in $B$. pseudomallei isolates from outside Australia. ${ }^{97}$ Whether melioidosis encephalomyelitis is truly limited both to genetically restricted strains of $B$. pseudomallei and in geographical location has implications for vaccine development as melioidosis encephalomyelitis often occurs in healthy people without the classical risk factors.

Occasionally, the primary presentation of melioidosis is septic arthritis or osteomyelitis, or one or both of these can develop after the patient has presented with another primary diagnosis, usually pneumonia. ${ }^{112}$ Also well recognized, whatever the clinical presentation, are abscesses in internal organs, especially spleen, kidney, prostate, and liver. That liver abscesses are also much more common in Thailand than in Australia may again reflect ingestion of $B$. pseudomalleicontaminated water from unchlorinated water sources.

Unusual foci of melioidosis infection described in case reports or case series include mycotic aneurysms, lymphadenitis resembling tuberculosis, mediastinal masses, pericardial collections, and pancreatitis. 


\section{Diagnosis: Culture of B. Pseudomallei Remains the Gold Standard}

Melioidosis must be considered in febrile patients in or returning from endemic regions to enable appropriate samples to be tested. Environmental recreational or occupational exposure history should prompt heightened vigilance, as should the presence of risk factors, most importantly diabetes. Isolation of B. pseudomallei by culture is currently the diagnostic gold standard, but can take up to 7 days for culture and confirmation. Serologic testing with indirect hemagglutination or various enzyme-linked immunosorbent assays ${ }^{113}$ without culture confirmation is considered inadequate to confirm a diagnosis because of the background seropositivity rates in those living in endemic locations. ${ }^{60}$ Furthermore, in acute septicemic melioidosis early serology is initially negative in up to half of cases, but repeat testing often shows seroconversion. ${ }^{114}$ Nevertheless, positive serology in a tourist returned from visiting an endemic area is useful in supporting the possibility of melioidosis, but definitive diagnosis still requires a positive culture.

B. pseudomallei readily grows in commercially available blood culture media, but it is not unusual for laboratories in nonendemic locations to misidentify the bacterium as a Pseudomonas or other Burkholderia species or to discount it as a contaminant, with some commercial identification systems being poor at identifying B. pseudomallei. ${ }^{115,116}$ Until they develop more comprehensive profile databases, caution is also required with identification of $B$. pseudomallei using the evolving matrix-assisted laser desorption ionization-time of flight mass spectrometry (MALDI-TOF MS) systems. ${ }^{117}$ Urine cultures and cultures from nonsterile sites increase the likelihood of diagnosis; the rate of successful diagnosis is increased if sputum, ulcer or skin lesion swabs, throat and rectal swabs are placed into Ashdown broth, a colistin-containing liquid transport medium that facilitates the selective growth of $B$. pseudomallei, or directly plated onto Ashdown agar which contains gentamicin or a commercial B. cepacia medium. ${ }^{118,119}$

B. pseudomallei can be identified from cultures by combining the commercial API 20NE or 20E biochemical kit profile with a simple screening system involving the Gram stain (gram-negative bacilli with characteristic bipolar staining with a "safety pin" appearance), oxidase reaction, typical growth characteristics, and resistance to certain antibiotics. ${ }^{120}$ Alternatively, flagged blood cultures or bacterial colonies on culture plates can now rapidly and accurately be identified using real-time PCR targeting the B. pseudomallei type III secretion system (TTS1) gene cluster ${ }^{121}$ or using various locally developed antigen detection systems that are not widely available, such as $B$. pseudomallei-specific latex agglutination and immunofluorescence. ${ }^{122,123}$

Direct real-time PCR assays of clinical samples have been trialed but, while providing a more rapid diagnosis, they have to date been less sensitive than blood cultures for detecting bacteremic melioidosis. ${ }^{124-126}$ Rapid immunofluorescence microscopy of pus, sputum, and urine has been useful in Thailand for rapid diagnosis but is not generally available elsewhere. ${ }^{127}$ Most recently a rapid point-of-care antigen detection test using a dipstick lateral flow immunoassay
(LFI) with a monoclonal antibody specifically targeting the B. pseudomallei capsular polysaccharide has shown promise when tested on sputum and pus. ${ }^{128}$ This LFI is, however, also less sensitive than culture for blood samples.

Irrespective of clinical presentation, chest X-ray and imaging of the abdomen and pelvis is recommended for all patients with melioidosis to assess for abscesses in internal organs. While abdominopelvic CT scanning is required for all postpubertal males to best exclude prostate abscesses, abdominal ultrasound is an alternative in children and females to avoid radiation exposure.

\section{Treatment: A Strong Evidence Base but New Directions Ahead?}

B. pseudomallei is characteristically resistant to penicillin, ampicillin, first- and second-generation cephalosporins, gentamicin, tobramycin, streptomycin, macrolides and polymyxins. Before 1989, "conventional therapy" for melioidosis consisted of a combination of chloramphenicol, trimethoprim-sulfamethoxazole, doxycycline, and sometimes kanamycin, given for 6 weeks to 6 months. ${ }^{4}$ There are also historical reports of the successful use of trimethoprimsulfamethoxazole alone and tetracycline or doxycycline alone. These conventional antibiotics are bacteriostatic rather than bactericidal, and in vitro studies have shown various combinations to be antagonistic.

B. pseudomallei is susceptible to various newer $\beta$-lactam antibiotics, especially ceftazidime, imipenem, meropenem, piperacillin, amoxicillin-clavulanate, ceftriaxone, and cefotaxime, with various degrees of bactericidal activity. The current recommendations for therapy of melioidosis are informed by a series of consecutive, large, elegant randomized comparative studies undertaken in northeast Thailand since 1986. A recent review of the treatment and prophylaxis of melioidosis comprehensively summarizes these studies and the historical and recent literature. ${ }^{129}$ In addition, formal guidelines have been published by the United States Centers for Disease Control and Prevention following a 2010 workshop with global expertise and representation, updating prior consensus guidelines. ${ }^{130,131}$ - Table 1 summarizes the current recommendations for therapy of melioidosis, ${ }^{1,129}$ - Table 2 provides dosing recommendations for patients with renal impairment, ${ }^{105}$ and - Table 3 shows the Darwin recommendations for duration of intravenous and oral phases of therapy based on the clinical presentation.

The most important clinical trial for melioidosis was an open-label randomized trial in Thailand comparing ceftazidime $(120 \mathrm{mg} / \mathrm{kg} / \mathrm{d})$ with conventional therapy. ${ }^{132}$ That study showed that ceftazidime is associated with a $50 \%$ lower overall mortality in severe melioidosis and from 1990 ceftazidime became the drug of choice for initial intensive therapy for melioidosis. Subsequently, high-dose imipenem was shown in another comparative trial from Thailand to be at least as effective as ceftazidime for severe melioidosis, with no differences in mortality between the groups but with fewer treatment failures in those given imipenem. ${ }^{133}$ The carbapenems imipenem and meropenem have the lowest minimum inhibitory concentrations against B. pseudomallei, 
Table 1 Therapy for melioidosis

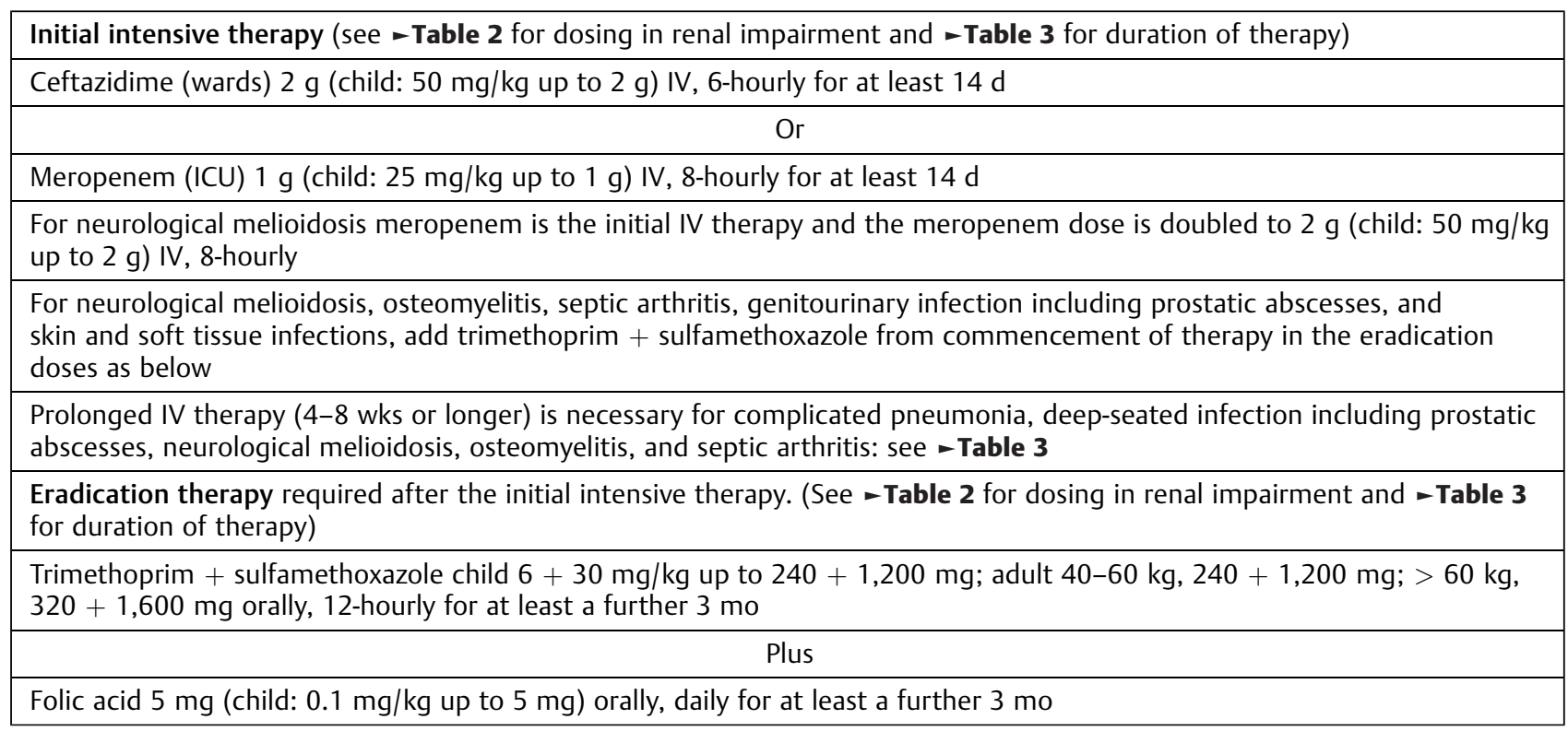

Abbreviations: ICU, intensive care unit; IV, intravenously.

Table 2 Adult treatment dosing in renal impairment

\begin{tabular}{|c|c|c|c|c|c|c|}
\hline & \multicolumn{3}{|c|}{ Dose adjustment by eGFR - CLcr (mL/min) ${ }^{a}$} & \multicolumn{3}{|c|}{ Dose adjustment for dialysis } \\
\hline & $31-50$ & $15-30$ & $<15$ & HD & CAPD & CRRT \\
\hline Ceftazidime & $\begin{array}{l}\text { Up to } 60 \mathrm{~kg} 1 \mathrm{~g} \\
\text { 8-hourly } \\
\text { Over } 60 \mathrm{~kg} 2 \mathrm{~g} \\
\text { 8-hourly }\end{array}$ & $\begin{array}{l}\text { Up to } 60 \mathrm{~kg} 1 \mathrm{~g} \\
\text { 12-hourly } \\
\text { Over } 60 \mathrm{~kg} 2 \mathrm{~g} \\
\text { 12-hourly }\end{array}$ & $\begin{array}{l}\text { Up to } 60 \mathrm{~kg} 1 \mathrm{~g} \\
\text { 24-hourly } \\
\text { Over } 60 \mathrm{~kg} 2 \mathrm{~g} \\
\text { 24-hourly }\end{array}$ & $\begin{array}{l}\text { As for eGFR } \\
<15 \text {, dose } \\
\text { after dialysis }\end{array}$ & $\begin{array}{l}\text { As for eGFR } \\
<15 \text { (if intra- } \\
\text { venous route is } \\
\text { inconvenient, } \\
\text { can administer } \\
\text { intraperitoneal- } \\
\text { ly with dwell } \\
\text { time of > } 6 \text { h } \\
\text { and } 25 \% \text { extra } \\
\text { dose) }\end{array}$ & $2 \mathrm{~g}$ 12-hourly \\
\hline Meropenem & $1 \mathrm{~g}$ 12-hourly & $1 \mathrm{~g}$ 12-hourly & $1 \mathrm{~g}$ 24-hourly & $\begin{array}{l}\text { As for eGFR } \\
<15 \text {, dose af- } \\
\text { ter dialysis }\end{array}$ & $\begin{array}{l}\text { As for } \\
\text { eGFR }<15\end{array}$ & $1 \mathrm{~g}$ 8-hourly \\
\hline $\mathrm{TMP}+\mathrm{SMX}$ & $\begin{array}{l}\text { Up to } 60 \mathrm{~kg} \\
240+1,200 \\
\text { mg } 12 \text {-hourly } \\
\text { Over } 60 \mathrm{~kg} \\
320+1,600 \\
\text { mg } 12 \text {-hourly }\end{array}$ & $\begin{array}{l}\text { Up to } 60 \mathrm{~kg} \\
240+1,200 \\
\text { mg } 24 \text {-hourly } \\
\text { Over } 60 \mathrm{~kg} \\
320+1,600 \\
\text { mg } 24 \text {-hourly }\end{array}$ & $\begin{array}{l}\text { Up to } 60 \mathrm{~kg} \\
240+1,200 \\
\text { mg } 24 \text {-hourly } \\
\text { Over } 60 \mathrm{~kg} \\
320+1,600 \\
\text { mg } 24 \text {-hourly }\end{array}$ & $\begin{array}{l}\text { As for eGFR } \\
<15 \text {, dose af- } \\
\text { ter dialysis }\end{array}$ & $\begin{array}{l}\text { As for } \\
\text { eGFR }<15\end{array}$ & $\begin{array}{l}\text { As for eGFR } \\
15-30\end{array}$ \\
\hline
\end{tabular}

Abbreviations: CAPD, chronic ambulatory peritoneal dialysis; CLcr, creatinine clearance, CRRT, continuous renal replacement therapy; eGFR, estimated glomerular filtration rate; HD, hemodialysis.

${ }^{\mathrm{a}}$ Creatinine clearance is calculated by Cockroft-Gault method $(140-$ age $[\mathrm{y}] \times$ ideal body weight $\times 0.85$ [if female]/0.814 $\times$ serum creatinine $[\mu \mathrm{mol} / \mathrm{L}] \times 72$ )

Recommend to use ideal body weight for weight-based dose calculation.

${ }^{\mathrm{b}} \mathrm{TMP}+\mathrm{SMX}$ : trimethoprim + sulfamethoxazole. Folic acid $5 \mathrm{mg}$ daily is added for the duration of therapy.

and in vitro time-kill studies to measure the rate of bacterial killing have shown the carbapenems to perform better against $B$. pseudomallei than ceftazidime. ${ }^{134,135}$ Observational data from Australia suggested that meropenem produces better outcomes in severe melioidosis than ceftazidime, resulting in the recommendation that meropenem be used as the drug of choice for severe melioidosis with septic shock. $^{136}$
Nevertheless, there is no evidence that ceftazidime is inferior to meropenem for patients with melioidosis who are not critically ill and ceftazidime remains the drug of choice for initial therapy for most patients. Furthermore, ceftazidime infusions ( 6 g over 24 hours, adult dose) through a peripherally inserted central catheter using an elastomeric infusion device (Baxter, Sydney, Australia) have enabled early hospital discharge for in-home therapy. ${ }^{137}$ Meropenem is not 
Table 3 Melioidosis treatment duration guideline

\begin{tabular}{|l|l|l|}
\hline Clinical focus & $\begin{array}{l}\text { Minimum intensive intravenous } \\
\text { phase duration (wk) }\end{array}$ & Eradication oral phase duration (mo) \\
\hline Skin abscess & 2 & 3 \\
\hline $\begin{array}{l}\text { Bacteremia with no } \\
\text { focus }\end{array}$ & 2 & 3 \\
\hline Pneumonia & $2-4$ & 3 \\
\hline $\begin{array}{l}\text { Prostatic abscess, septic } \\
\text { arthritis and organ or } \\
\text { deep-seated tissue } \\
\text { collection }\end{array}$ & 4 & 3 \\
\hline Osteomyelitis & 6 & 6 \\
\hline $\begin{array}{l}\text { Central nervous system } \\
\text { infection }\end{array}$ & 8 & 6 \\
\hline
\end{tabular}

a Use clinical judgment to guide prolongation of intensive phase if improvement is slow or if blood cultures remain positive at 7 days.

${ }^{b}$ Intensive phase duration is timed from date of most recent drainage of collection (e.g., prostatic abscess or joint washout) where culture of the drainage specimen grew $B$. pseudomallei or where no specimen was sent for culture; clock is not reset if drainage specimen is culture negative.

adequately stable at ambient temperature in the tropics for such in-home therapy.

Antimicrobial therapy is separated into the initial intensive phase and the subsequent eradication phase.

\section{Intensive Phase}

Intravenous ceftazidime or meropenem is used and the duration of initial intensive therapy should be a minimum of 10 to 14 days, with longer treatment required for critically ill patients, or for extensive pulmonary disease, deep-seated collections or organ abscesses, osteomyelitis, septic arthritis, and neurologic melioidosis. The therapeutic response can be slow, with median time to defervescence up to 9 days, and longer times seen in those with deep-seated abscesses.

Whether trimethoprim-sulfamethoxazole added to ceftazidime is superior to ceftazidime alone has been studied in two randomized controlled trials in Thailand. ${ }^{138}$ Although the addition of trimethoprim-sulfamethoxazole conferred no survival benefit, ${ }^{139}$ the excellent tissue penetration of trimethoprim-sulfamethoxazole is the rationale for recommending combination therapy in neurologic, cutaneous, bone, joint, and prostatic melioidosis.

A large study from Thailand showed that primary resistance to ceftazidime can occur but is exceedingly rare $(0.05 \%){ }^{140}$ Acquired ceftazidime resistance (occurring subsequent to treatment commencement) remains very uncommon but has been well described and the genetic basis of several resistance mechanisms involved has been elucidated, including point mutations and gene deletions affecting the class A $\beta$-lactamase, PenA. ${ }^{141,142}$ Primary or acquired resistance to meropenem in $B$. pseudomallei has not been seen in Australia and is yet to be adequately documented elsewhere. $^{143}$

\section{Eradication Phase}

After initial intensive therapy, subsequent eradication therapy has been considered necessary for preventing recrudescence or later relapses of melioidosis. Again the regimens and duration have been studied in a series of trials in Thailand, the most recent showing that adding doxycycline to trimethoprim-sulfamethoxazole confers no advantage over use of trimethoprim-sulfamethoxazole alone. ${ }^{144}$ Initial reports of primary resistance to trimethoprim-sulfamethoxazole in over $10 \%$ of $B$. pseudomallei isolates from Thailand and other Southeast Asian countries have recently been shown to likely be incorrect and this reflects the difficulties of assessing susceptibility to trimethoprim-sulfamethoxazole using disc diffusion rather than the validated $\mathrm{E}$ testing. ${ }^{145}$ Primary resistance to trimethoprim-sulfamethoxazole in isolates from Australia is exceptionally rare (2/234 resistant from consecutive cases, with only low level resistance in these 2). ${ }^{143}$ Therefore, trimethoprim-sulfamethoxazole should generally be considered the initial eradication agent of choice for melioidosis globally.

Amoxicillin-clavulanate has been used as an alternative in children and in pregnancy in some locations in Thailand but in Australia trimethoprim-sulfamethoxazole is the preferred eradication therapy for children. ${ }^{68}$ Acquired resistance is well documented when amoxicillin-clavulanate or doxycycline is used and although less common, acquired resistance is also occasionally seen for trimethoprim-sulfamethoxazole. ${ }^{146-150}$

Dosing recommendations for trimethoprim-sulfamethoxazole and amoxicillin-clavulanate are based on modeling and empirical experience and are higher than the standard doses generally used with these antibiotics. ${ }^{151,152}$ It is not uncommon for adverse effects to occur with the prolonged course of trimethoprim-sulfamethoxazole required, with rash, gastrointestinal symptoms, hyperkalemia, and rising creatinine sometimes requiring dose modification or a switch to the alternatives of doxycycline or amoxicillin-clavulanate. Desensitization is worth attempting for nonsevere skin reactions attributed to trimethoprim-sulfamethoxazole.

\section{Duration of Intensive Phase, Eradication Phase, and Future Directions}

Studies from Thailand have shown that failure of eradication therapy is associated with poor compliance with 
therapy, more severe disease (bacteremia and multifocal disease) and duration of eradication therapy of less than 8 weeks. $^{70,153,154}$ This is the basis of the current recommendations that the duration of the eradication phase be for 3 to 6 months.

Over the last decade, however, the duration of intravenous therapy in patients treated at Royal Darwin Hospital has increased, with local guidelines defining the minimum recommended duration of intravenous therapy based on the clinical presentation (-Table 3 ). The median duration of intravenous therapy for patients is now around 4 weeks. Of the 410 patients admitted in the 9 years from October 2003 to September 2012 , only $5(1.2 \%)$ have relapsed, which is significantly lower than in previous years $(6.4 \% ; p<0.001)$ 85 and also lower than that reported from Thailand (9.7\%). ${ }^{70}$ This is despite patients commonly ceasing the eradication therapy early, frequently missing doses or not taking it at all. The substantial decline in relapsed melioidosis in the Darwin study despite the poor adherence to eradication therapy has been attributed to the prolongation of the intravenous phase for many patients and raises the possibility of future guidelines based solely intravenous therapy and without the need for a prolonged eradication phase.

Recent case series do support the potential role for therapy with oral trimethoprim-sulfamethoxazole for 3 months without a preceding intravenous phase for selected patients with localized cutaneous disease. ${ }^{68,103,155}$ Oral therapy alone should be restricted to healthy patients with no underlying risk factors who are systemically well and where dissemination or infection at other sites including lymph node involvement has been excluded by a full melioidosis workup.

\section{Other Therapeutic Aspects}

Prostatic abscesses usually require drainage done under CT or portable transrectal ultrasound guidance, which can be performed in ventilated patients. ${ }^{112}$ Transurethral resection, is reserved for failures of the simpler procedures. Septic arthritis usually requires operative drainage and washout, sometimes necessitating multiple theater visits, especially when there is adjacent osteomyelitis. While early long bone osteomyelitis without abscess formation and vertebral osteomyelitis without epidural abscess may not require debridement, aggressive surgical debridement of necrotic bone is commonly necessary when diagnosis and appropriate antibiotic therapy are delayed. Mycotic aneurysms are increasingly recognized as occurring in melioidosis and require urgent surgery, often with insertion of prosthetic vascular grafts. For those with prosthetic grafts lifelong suppressive therapy with trimethoprim-sulfamethoxazole may be indicated. In a recent case of melioidosis mycotic abdominal aortic aneurysm in a traveler returned to Denmark from a vacation in Thailand, an autologous deep vein graft was used, allowing cessation of therapy after 6 months total therapy. ${ }^{32}$ Surgical drainage of large abscesses is indicated, but this is usually not necessary or possible for multiple small abscesses in the spleen and liver. Other internal collections frequently resolve with medical therapy and rarely need to be drained.
State-of-the-art intensive care management has resulted in a substantial decrease in mortality in patients with melioidosis septic shock. Together with resources for timely laboratory diagnosis of melioidosis and availability of ceftazidime, what is critical is access to an adequately staffed intensive care facility with capability of invasive monitoring, quality airway management and ventilation and inotropic support and renal replacement therapy. ${ }^{156}$ In December 1998 empirical use of granulocyte colony-stimulating factor (G-CSF) in patients with strictly defined melioidosis septic shock was commenced in the intensive care unit at Royal Darwin Hospital in an attempt to counteract the functional neutrophil defects thought to be critical in the pathogenesis of severe melioidosis. Observational data showed a significant improvement in survival with G-CSF. ${ }^{157}$ Nevertheless, concomitant improvements in other aspects of patient management were confounding factors in that study. A randomized controlled trial in Thailand showed no survival benefit of GCSF in that location where there are limited intensive care resources, although survival was longer in the G-CSF group. ${ }^{158}$ Given the importance of immune function in the pathogenesis of melioidosis, patients with melioidosis will be a specific group that is worthy of study as other immunemodulating therapies become available for use in patients with sepsis.

Various newer antimicrobials have been tested in vivo and in animal models, with none to date likely to replace ceftazidime or meropenem. ${ }^{129}$ Doripenem has minimum inhibitory concentrations similar to meropenem but ertapenem, tigecycline, and moxifloxacin appear to have limited in vitro activity. ${ }^{159}$ Other potential novel therapeutic approaches for melioidosis have recently been extensively reviewed. ${ }^{160-162}$

Development of a melioidosis vaccine has been heavily funded in recent years because of the biothreat potential of $B$. pseudomallei and for military purposes. A vaccine could also have substantial benefits for those living in endemic regions and for commercial livestock, although cost will be a major impediment to availability. Preliminary studies have included various conjugate, live attenuated, and heterologous vaccine candidates, with none published to date conferring sterilizing immunity. 163,164

\section{Concluding Remarks}

B. pseudomallei and melioidosis have generated intense and increasing interest over the last two decades, with many high quality studies spanning molecular genomics, cutting edge immunology and cell biology, animal challenge studies, clinical microbiology and new diagnostics, large descriptive epidemiological and clinical studies and large randomized controlled studies of antimicrobials. Mortality is now under $10 \%$ overall in locations with resources for rapid diagnosis, early implementation of best antibiotics, and state-of-the-art intensive care facilities for managing severe sepsis. However, such resources are just not available or are extremely limited in many of the regions where melioidosis is endemic. Around 25 years after Prof. Nick White and his colleagues in Thailand showed a halving of mortality from melioidosis with ceftazidime, that antibiotic is still not available in much of 
Cambodia, where mortality from melioidosis is thought to still be over $50 \%$.

In the same world but under very different circumstances we can ask "Is melioidosis as real a biothreat potential as the research funding would suggest?" While $B$. pseudomallei can be aerosolized and animal inhalation challenge studies show high lethality, the paradox is that fatal melioidosis is extremely unlikely from natural infection in a healthy person, provided early diagnosis and therapy are available. Melioidosis could almost be classified as another opportunistic infection. Nevertheless, while the clinical outcomes in healthy human hosts of inhalation of a high bacterial load from a deliberate release of $B$. pseudomallei remain uncertain, there are enough diabetics and others with risk factors for melioidosis in major urban centers to make a biothreat scenario a potential medical disaster that could rapidly overwhelm intensive care facilities.

\section{Acknowledgments}

I would like to thank all my clinical and laboratory colleagues at the Royal Darwin Hospital and the Menzies School of Health Research for support with the Darwin melioidosis studies, which have also been supported by project grants from the Australian National Health and Medical Research Council. Also, the global melioidosis research community for collegiality and collaboration over many years.

\section{References}

1 Wiersinga WJ, Currie BJ, Peacock SJ. Melioidosis. N Engl J Med 2012;367(11):1035-1044

2 White NJ. Melioidosis. Lancet 2003;361(9370):1715-1722

3 Cheng AC, Currie BJ. Melioidosis: epidemiology, pathophysiology, and management. Clin Microbiol Rev 2005;18(2):383-416

4 Leelarasamee A, Bovornkitti S. Melioidosis: review and update. Rev Infect Dis 1989;11(3):413-425

5 Vandamme P, Dawyndt P. Classification and identification of the Burkholderia cepacia complex: Past, present and future. Syst Appl Microbiol 2011;34(2):87-95

6 Compant S, Nowak J, Coenye T, Clément C, Ait Barka E. Diversity and occurrence of Burkholderia spp. in the natural environment. FEMS Microbiol Rev 2008;32(4):607-626

7 Gee JE, Glass MB, Novak RT, et al. Recovery of a Burkholderia thailandensis-like isolate from an Australian water source. BMC Microbiol 2008;8:54

8 Liu X, Biswas S, Berg MG, et al. Genomics-guided discovery of thailanstatins A, B, and C As pre-mRNA splicing inhibitors and antiproliferative agents from Burkholderia thailandensis MSMB43. J Nat Prod 2013;76(4):685-693

9 Coenye T, Spilker T, Van Schoor A, LiPuma JJ, Vandamme P. Recovery of Burkholderia cenocepacia strain PHDC from cystic fibrosis patients in Europe. Thorax 2004;59(11):952-954

10 Whitmore A, Krishnaswami CS. An account of the discovery of a hitherto underscribed infective disease occurring among the population of Rangoon. Ind Med Gaz 1912;47:262-267

11 Stanton AT, Fletcher W. Melioidosis: a new disease of the tropics. Trans Fourth Congr Far East Assoc Trop Med 1921;2:196-198

12 Godoy D, Randle G, Simpson AJ, et al. Multilocus sequence typing and evolutionary relationships among the causative agents of melioidosis and glanders, Burkholderia pseudomallei and Burkholderia mallei. J Clin Microbiol 2003;41(5):2068-2079

13 Holden MT, Titball RW, Peacock SJ, et al. Genomic plasticity of the causative agent of melioidosis, Burkholderia pseudomallei. Proc Natl Acad Sci U S A 2004;101(39):14240-14245

14 Nierman WC, DeShazer D, Kim HS, et al. Structural flexibility in the Burkholderia mallei genome. Proc Natl Acad Sci U S A 2004; 101(39):14246-14251

15 Deitchman S, Sokas R. Glanders in a military research microbiologist. N Engl J Med 2001;345(22):1644

16 Currie BJ, Ward L, Cheng AC. The epidemiology and clinical spectrum of melioidosis: 540 cases from the 20 year Darwin prospective study. PLoS Negl Trop Dis 2010;4(11):e900

17 Wheelis M. First shots fired in biological warfare. Nature 1998; 395(6699):213

18 Butler D. Viral research faces clampdown. Nature 2012; 490(7421):456

19 Dance DA. Melioidosis: the tip of the iceberg? Clin Microbiol Rev 1991;4(1):52-60

20 Leelarasamee A. Melioidosis in Southeast Asia. Acta Trop 2000; 74(2-3):129-132

21 Limmathurotsakul D, Wongratanacheewin S, Teerawattanasook $\mathrm{N}$, et al. Increasing incidence of human melioidosis in Northeast Thailand. Am J Trop Med Hyg 2010;82(6):1113-1117

22 Chaowagul W, White NJ, Dance DA, et al. Melioidosis: a major cause of community-acquired septicemia in northeastern Thailand. J Infect Dis 1989;159(5):890-899

23 Parameswaran U, Baird RW, Ward LM, Currie BJ. Melioidosis at Royal Darwin Hospital in the big 2009-2010 wet season: comparison with the preceding 20 years. Med J Aust 2012;196(5):345-348

24 Currie BJ, Dance DA, Cheng AC. The global distribution of Burkholderia pseudomallei and melioidosis: an update. Trans R Soc Trop Med Hyg 2008;102(Suppl 1):S1-S4

25 Inglis TJ, Rolim DB, Sousa AdeQ. Melioidosis in the Americas. Am J Trop Med Hyg 2006;75(5):947-954

26 Doker TJ, Sharp TM, Rivera-Garcia B, et al. Contact Investigation of Melioidosis Cases Reveals Regional Endemicity in Puerto Rico. Clin Infect Dis 2014

27 Garin B, Djaomazala I, Dubois-Cauwelaert N, et al; Mahafaly. Autochthonous melioidosis in humans, Madagascar, 2012 and 2013. Emerg Infect Dis 2014;20(10):1739-1741

28 Katangwe T, Purcell J, Bar-Zeev N, et al. Human melioidosis, Malawi, 2011. Emerg Infect Dis 2013;19(6):981-984

29 Kaestli M, Schmid M, Mayo M, et al. Out of the ground: aerial and exotic habitats of the melioidosis bacterium Burkholderia pseudomallei in grasses in Australia. Environ Microbiol 2012;14(8): 2058-2070

30 Pearson T, Giffard P, Beckstrom-Sternberg S, et al. Phylogeographic reconstruction of a bacterial species with high levels of lateral gene transfer. BMC Biol 2009;7:78

31 Gee JE, Allender CJ, Tuanyok A, Elrod MG, Hoffmaster AR. Burkholderia pseudomallei type $G$ in Western Hemisphere. Emerg Infect Dis 2014;20(4):682-684

32 Bodilsen J, Vammen S, Fuursted K, Hjort U. Mycotic aneurysm caused by Burkholderia pseudomallei in a previously healthy returning traveller. BMJ Case Rep 2014 Sep 22;2014. pii: bcr2013202824. doi: 10.1136/bcr-2013-202824

33 Currie BJ. Melioidosis: an important cause of pneumonia in residents of and travellers returned from endemic regions. Eur Respir J 2003;22(3):542-550

34 Doker TJ, Quinn CL, Salehi ED, et al; Melioidosis Investigation Team. Fatal Burkholderia pseudomallei infection initially reported as a Bacillus species, Ohio, 2013. Am J Trop Med Hyg 2014;91(4):743-746

35 Stewart T, Engelthaler DM, Blaney DD, et al. Epidemiology and investigation of melioidosis, Southern Arizona. Emerg Infect Dis 2011;17(7):1286-1288 
36 Engelthaler DM, Bowers J, Schupp JA, et al. Molecular investigations of a locally acquired case of melioidosis in Southern AZ, USA. PLoS Negl Trop Dis 2011;5(10):e1347

37 Limmathurotsakul D, Kanoksil M, Wuthiekanun V, et al. Activities of daily living associated with acquisition of melioidosis in northeast Thailand: a matched case-control study. PLoS Negl Trop Dis 2013;7(2):e2072

38 Inglis TJ, Garrow SC, Henderson M, et al. Burkholderia pseudomallei traced to water treatment plant in Australia. Emerg Infect Dis 2000;6(1):56-59

39 Currie BJ, Mayo M, Anstey NM, Donohoe P, Haase A, Kemp DJ. A cluster of melioidosis cases from an endemic region is clonal and is linked to the water supply using molecular typing of Burkholderia pseudomallei isolates. Am J Trop Med Hyg 2001;65(3): 177-179

40 Ralph A, McBride J, Currie BJ. Transmission of Burkholderia pseudomallei via breast milk in northern Australia. Pediatr Infect Dis J 2004;23(12):1169-1171

41 Thatrimontrichai A, Maneenil G. Neonatal melioidosis: systematic review of the literature. Pediatr Infect Dis J 2012;31(11): 1195-1197

42 Choy JL, Mayo M, Janmaat A, Currie BJ. Animal melioidosis in Australia. Acta Trop 2000;74(2-3):153-158

43 Cheng AC, Jacups SP, Gal D, Mayo M, Currie BJ. Extreme weather events and environmental contamination are associated with case-clusters of melioidosis in the Northern Territory of Australia. Int J Epidemiol 2006;35(2):323-329

44 Lo TJ, Ang LW, James L, Goh KT. Melioidosis in a tropical city state, Singapore. Emerg Infect Dis 2009;15(10):1645-1647

45 Currie BJ, Jacups SP. Intensity of rainfall and severity of melioidosis, Australia. Emerg Infect Dis 2003;9(12):1538-1542

46 Ko WC, Cheung BM, Tang HJ, et al. Melioidosis outbreak after typhoon, southern Taiwan. Emerg Infect Dis 2007;13(6):896-898

47 Chen YL, Yen YC, Yang CY, et al. The concentrations of ambient Burkholderia pseudomallei during typhoon season in endemic area of melioidosis in Taiwan. PLoS Negl Trop Dis 2014;8(5): e2877

48 Currie BJ, Fisher DA, Howard DM, et al. The epidemiology of melioidosis in Australia and Papua New Guinea. Acta Trop 2000; 74(2-3):121-127

49 Allworth AM. Tsunami lung: a necrotising pneumonia in survivors of the Asian tsunami. Med J Aust 2005;182(7):364

50 Athan E, Allworth AM, Engler C, Bastian I, Cheng AC. Melioidosis in tsunami survivors. Emerg Infect Dis 2005;11(10): 1638-1639

51 Chierakul W, Winothai W, Wattanawaitunechai C, et al. Melioidosis in 6 tsunami survivors in southern Thailand. Clin Infect Dis 2005;41(7):982-990

52 Howe C, Sampath A, Spotnitz M. The pseudomallei group: a review. J Infect Dis 1971;124(6):598-606

53 Amadasi S, Dal Zoppo S, Bonomini A, et al. A Case of Melioidosis Probably Acquired by Inhalation of Dusts During a Helicopter Flight in a Healthy Traveler Returning From Singapore. J Travel Med 2014

54 Jeddeloh JA, Fritz DL, Waag DM, Hartings JM, Andrews GP. Biodefense-driven murine model of pneumonic melioidosis. Infect Immun 2003;71(1):584-587

55 West TE, Myers ND, Liggitt HD, Skerrett SJ. Murine pulmonary infection and inflammation induced by inhalation of Burkholderia pseudomallei. Int J Exp Pathol 2012;93(6):421-428

56 Nelson M, Dean RE, Salguero FJ, et al. Development of an acute model of inhalational melioidosis in the common marmoset (Callithrix jacchus). Int J Exp Pathol 2011;92(6):428-435

57 Titball RW, Russell P, Cuccui J, et al. Burkholderia pseudomallei: animal models of infection. Trans R Soc Trop Med Hyg 2008;102 (Suppl 1):S111-S116

58 Cheng AC, Currie BJ, Dance DA, et al. Clinical definitions of melioidosis. Am J Trop Med Hyg 2013;88(3):411-413
59 Cheng AC, Wuthiekanun V, Limmathurotsakul D, Chierakul W, Peacock SJ. Intensity of exposure and incidence of melioidosis in Thai children. Trans R Soc Trop Med Hyg 2008;102(Suppl 1): S37-S39

60 Wuthiekanun V, Chierakul W, Langa S, et al. Development of antibodies to Burkholderia pseudomallei during childhood in melioidosis-endemic northeast Thailand. Am J Trop Med Hyg 2006;74(6):1074-1075

61 James GL, Delaney B, Ward L, Freeman K, Mayo M, Currie BJ. Surprisingly low seroprevalence of Burkholderia pseudomallei in exposed healthy adults in the Darwin region of tropical Australia where melioidosis is highly endemic. Clin Vaccine Immunol 2013;20(5):759-760

62 Ashdown LR, Guard RW. The prevalence of human melioidosis in Northern Queensland. Am J Trop Med Hyg 1984;33(3):474-478

63 Clayton AJ, Lisella RS, Martin DG. Melioidosis: a serological survey in military personnel. Mil Med 1973;138(1):24-26

64 Currie BJ, Fisher DA, Anstey NM, Jacups SP. Melioidosis: acute and chronic disease, relapse and re-activation. Trans R Soc Trop Med Hyg 2000;94(3):301-304

65 Suputtamongkol Y, Hall AJ, Dance DA, et al. The epidemiology of melioidosis in Ubon Ratchatani, northeast Thailand. Int J Epidemiol 1994;23(5):1082-1090

66 Ngauy V, Lemeshev Y, Sadkowski L, Crawford G. Cutaneous melioidosis in a man who was taken as a prisoner of war by the Japanese during World War II. J Clin Microbiol 2005;43(2): 970-972

67 Kingston CW. Chronic or latent melioidosis. Med J Aust 1971; 2(12):618-621

68 McLeod C, Morris PS, Bauert PA, et al. Clinical presentation and medical management of melioidosis in children: a 24-year prospective study in the Northern Territory of Australia and review of the literature. Clin Infect Dis 2014

69 Suputtamongkol Y, Chaowagul W, Chetchotisakd P, et al. Risk factors for melioidosis and bacteremic melioidosis. Clin Infect Dis 1999;29(2):408-413

70 Limmathurotsakul D, Chaowagul W, Chierakul W, et al. Risk factors for recurrent melioidosis in northeast Thailand. Clin Infect Dis 2006;43(8):979-986

71 Currie BJ, Fisher DA, Howard DM, et al. Endemic melioidosis in tropical northern Australia: a 10-year prospective study and review of the literature. Clin Infect Dis 2000;31(4):981-986

72 Easton A, Haque A, Chu K, Lukaszewski R, Bancroft GJ. A critical role for neutrophils in resistance to experimental infection with Burkholderia pseudomallei. J Infect Dis 2007;195(1):99-107

73 Wiersinga WJ, van der Poll T, White NJ, Day NP, Peacock SJ. Melioidosis: insights into the pathogenicity of Burkholderia pseudomallei. Nat Rev Microbiol 2006;4(4):272-282

74 Wiersinga WJ, Wieland CW, Dessing MC, et al. Toll-like receptor 2 impairs host defense in gram-negative sepsis caused by Burkholderia pseudomallei (Melioidosis). PLoS Med 2007;4(7):e248

75 West TE, Myers ND, Chantratita N, et al. NLRC4 and TLR5 each contribute to host defense in respiratory melioidosis. PLoS Negl Trop Dis 2014;8(9):e3178

76 Myers ND, Chantratita N, Berrington WR, et al. The role of NOD2 in murine and human melioidosis. J Immunol 2014;192(1): 300-307

77 Nuntayanuwat S, Dharakul T, Chaowagul W, Songsivilai S. Polymorphism in the promoter region of tumor necrosis factor-alpha gene is associated with severe meliodosis. Hum Immunol 1999; 60(10):979-983

78 Chantratita N, Tandhavanant S, Myers ND, et al. Common TLR1 genetic variation is not associated with death from melioidosis, a common cause of sepsis in rural Thailand. PLoS ONE 2014;9(1): e83285

79 Chantratita N, Tandhavanant S, Myers ND, et al. Screen of whole blood responses to flagellin identifies TLR5 variation associated with outcome in melioidosis. Genes Immun 2014;15(2):63-71 
80 West TE, Chierakul W, Chantratita N, et al. Toll-like receptor 4 region genetic variants are associated with susceptibility to melioidosis. Genes Immun 2012;13(1):38-46

81 West TE, Chantratita N, Chierakul W, et al. Impaired TLR5 functionality is associated with survival in melioidosis. J Immunol 2013;190(7):3373-3379

82 Barnes JL, Warner J, Melrose W, et al. Adaptive immunity in melioidosis: a possible role for T cells in determining outcome of infection with Burkholderia pseudomallei. Clin Immunol 2004; 113(1):22-28

83 Ketheesan N, Barnes JL, Ulett GC, et al. Demonstration of a cellmediated immune response in melioidosis. J Infect Dis 2002; 186(2):286-289

84 Chierakul W, Wuthiekanun V, Chaowagul W, et al. Short report: disease severity and outcome of melioidosis in HIV coinfected individuals. Am J Trop Med Hyg 2005;73(6):1165-1166

85 Sarovich DS, Ward L, Price EP, et al. Recurrent melioidosis in the Darwin Prospective Melioidosis Study: improving therapies mean that relapse cases are now rare. J Clin Microbiol 2014; 52(2):650-653

86 Tuanyok A, Auerbach RK, Brettin TS, et al. A horizontal gene transfer event defines two distinct groups within Burkholderia pseudomallei that have dissimilar geographic distributions. J Bacteriol 2007;189(24):9044-9049

87 Tuanyok A, Leadem BR, Auerbach RK, et al. Genomic islands from five strains of Burkholderia pseudomallei. BMC Genomics 2008; 9:566

88 Reckseidler-Zenteno SL, DeVinney R, Woods DE. The capsular polysaccharide of Burkholderia pseudomallei contributes to survival in serum by reducing complement factor $\mathrm{C} 3 \mathrm{~b}$ deposition. Infect Immun 2005;73(2):1106-1115

89 DeShazer D, Brett PJ, Woods DE. The type II O-antigenic polysaccharide moiety of Burkholderia pseudomallei lipopolysaccharide is required for serum resistance and virulence. Mol Microbiol 1998;30(5):1081-1100

90 Stevens MP, Wood MW, Taylor LA, et al. An Inv/Mxi-Spa-like type III protein secretion system in Burkholderia pseudomallei modulates intracellular behaviour of the pathogen. Mol Microbiol 2002;46(3):649-659

91 Burtnick MN, Brett PJ, Harding SV, et al. The cluster 1 type VI secretion system is a major virulence determinant in Burkholderia pseudomallei. Infect Immun 2011;79(4):1512-1525

92 Ulrich RL, Deshazer D, Brueggemann EE, Hines HB, Oyston PC, Jeddeloh JA. Role of quorum sensing in the pathogenicity of Burkholderia pseudomallei. J Med Microbiol 2004;53(Pt 11): 1053-1064

93 Cruz-Migoni A, Hautbergue GM, Artymiuk PJ, et al. A Burkholderia pseudomallei toxin inhibits helicase activity of translation factor eIF4A. Science 2011;334(6057):821-824

94 Chantratita N, Tandhavanant S, Wikraiphat C, et al. Proteomic analysis of colony morphology variants of Burkholderia pseudomallei defines a role for the arginine deiminase system in bacterial survival. J Proteomics 2012;75(3):1031-1042

95 Chantratita N, Wuthiekanun V, Boonbumrung K, et al. Biological relevance of colony morphology and phenotypic switching by Burkholderia pseudomallei. J Bacteriol 2007;189(3): 807-817

96 Ulett GC, Currie BJ, Clair TW, et al. Burkholderia pseudomallei virulence: definition, stability and association with clonality. Microbes Infect 2001;3(8):621-631

97 Sarovich DS, Price EP, Webb JR, et al. Variable virulence factors in Burkholderia pseudomallei (melioidosis) associated with human disease. PLoS ONE 2014;9(3):e91682

98 Achana V, Silpapojakul K, Thininta W, Kalnaowakul S. Acute Pseudomonas pseudomallei pneumonia and septicemia following aspiration of contaminated water: a case report. Southeast Asian J Trop Med Public Health 1985;16(3):500-504
99 Lee $\mathrm{N}, \mathrm{Wu} \mathrm{JL}$, Lee $\mathrm{CH}$, Tsai WC. Pseudomonas pseudomallei infection from drowning: the first reported case in Taiwan. J Clin Microbiol 1985;22(3):352-354

100 Meumann EM, Cheng AC, Ward L, Currie BJ. Clinical features and epidemiology of melioidosis pneumonia: results from a 21-year study and review of the literature. Clin Infect Dis 2012;54(3): 362-369

101 Gibney KB, Cheng AC, Currie BJ. Cutaneous melioidosis in the tropical top end of Australia: a prospective study and review of the literature. Clin Infect Dis 2008;47(5):603-609

102 Dance DA, Davis TM, Wattanagoon Y, et al. Acute suppurative parotitis caused by Pseudomonas pseudomallei in children. J Infect Dis 1989;159(4):654-660

103 Pagnarith Y, Kumar V, Thaipadungpanit J, et al. Emergence of pediatric melioidosis in Siem Reap, Cambodia. Am J Trop Med Hyg 2010;82(6):1106-1112

104 Morse LP, Moller CC, Harvey E, et al. Prostatic abscess due to Burkholderia pseudomallei: 81 cases from a 19-year prospective melioidosis study. J Urol 2009;182(2):542-547, discussion 547

105 Jabbar Z, Currie BJ. Melioidosis and the kidney. Nephrology (Carlton) 2013;18(3):169-175

106 Woods ML II, Currie BJ, Howard DM, et al. Neurological melioidosis: seven cases from the Northern Territory of Australia. Clin Infect Dis 1992;15(1):163-169

107 Currie BJ, Fisher DA, Howard DM, Burrow JN. Neurological melioidosis. Acta Trop 2000;74(2-3):145-151

108 Koszyca B, Currie BJ, Blumbergs PC. The neuropathology of melioidosis: two cases and a review of the literature. Clin Neuropathol 2004;23(5):195-203

109 Chadwick DR, Ang B, Sitoh YY, Lee CC. Cerebral melioidosis in Singapore: a review of five cases. Trans R Soc Trop Med Hyg 2002; 96(1):72-76

110 Owen SJ, Batzloff M, Chehrehasa F, et al. Nasal-associated lymphoid tissue and olfactory epithelium as portals of entry for Burkholderia pseudomallei in murine melioidosis. J Infect Dis 2009;199(12):1761-1770

111 St John JA, Ekberg JA, Dando SJ, et al. Burkholderia pseudomallei penetrates the brain via destruction of the olfactory and trigeminal nerves: implications for the pathogenesis of neurological melioidosis. MBio 2014;5(2):e00025

112 Morse LP, Smith J, Mehta J, Ward L, Cheng AC, Currie BJ. Osteomyelitis and septic arthritis from infection with Burkholderia pseudomallei: A 20-year prospective melioidosis study from northern Australia. J Orthop 2013;10(2):86-91

113 Cooper A, Williams NL, Morris JL, Norton RE, Ketheesan N, Schaeffer PM. ELISA and immuno-polymerase chain reaction assays for the sensitive detection of melioidosis. Diagn Microbiol Infect Dis 2013;75(2):135-138

114 Cheng AC, O'brien M, Freeman K, Lum G, Currie BJ. Indirect hemagglutination assay in patients with melioidosis in northern Australia. Am J Trop Med Hyg 2006;74(2):330-334

115 Lowe P, Engler C, Norton R. Comparison of automated and nonautomated systems for identification of Burkholderia pseudomallei. J Clin Microbiol 2002;40(12):4625-4627

116 Podin Y, Kaestli M, McMahon N, et al. Reliability of automated biochemical identification of Burkholderia pseudomallei is regionally dependent. J Clin Microbiol 2013;51(9):3076-3078

117 Cunningham SA, Patel R. Importance of using Bruker's securityrelevant library for Biotyper identification of Burkholderia pseudomallei, Brucella species, and Francisella tularensis. JClin Microbiol 2013;51(5):1639-1640

118 Peacock SJ, Chieng G, Cheng AC, et al. Comparison of Ashdown's medium, Burkholderia cepacia medium, and Burkholderia pseudomallei selective agar for clinical isolation of Burkholderia pseudomallei. J Clin Microbiol 2005;43(10):5359-5361

119 Wuthiekanun V, Dance DA, Wattanagoon Y, Supputtamongkol Y, Chaowagul W, White NJ. The use of selective media for the 
isolation of Pseudomonas pseudomallei in clinical practice. J Med Microbiol 1990;33(2):121-126

120 Dance DA, Wuthiekanun V, Naigowit P, White NJ. Identification of Pseudomonas pseudomallei in clinical practice: use of simple screening tests and API 20NE. J Clin Pathol 1989;42(6):645-648

121 Novak RT, Glass MB, Gee JE, et al. Development and evaluation of a real-time PCR assay targeting the type III secretion system of Burkholderia pseudomallei. J Clin Microbiol 2006;44(1):85-90

122 Amornchai P, Chierakul W, Wuthiekanun V, et al. Accuracy of Burkholderia pseudomallei identification using the API 20NE system and a latex agglutination test. J Clin Microbiol 2007; 45(11):3774-3776

123 Chantratita N, Tandhavanant S, Wongsuvan G, et al. Rapid detection of Burkholderia pseudomallei in blood cultures using a monoclonal antibody-based immunofluorescent assay. Am J Trop Med Hyg 2013;89(5):971-972

124 Kaestli M, Richardson LJ, Colman RE, et al. Comparison of TaqMan PCR assays for detection of the melioidosis agent Burkholderia pseudomallei in clinical specimens. J Clin Microbiol 2012;50(6): 2059-2062

125 Meumann EM, Novak RT, Gal D, et al. Clinical evaluation of a type III secretion system real-time PCR assay for diagnosing melioidosis. J Clin Microbiol 2006;44(8):3028-3030

126 Chantratita N, Meumann E, Thanwisai A, et al. Loop-mediated isothermal amplification method targeting the TTS1 gene cluster for detection of Burkholderia pseudomallei and diagnosis of melioidosis. J Clin Microbiol 2008;46(2):568-573

127 Wuthiekanun V, Desakorn V, Wongsuvan G, et al. Rapid immunofluorescence microscopy for diagnosis of melioidosis. Clin Diagn Lab Immunol 2005;12(4):555-556

128 Houghton RL, Reed DE, Hubbard MA, et al. Development of a prototype lateral flow immunoassay (LFI) for the rapid diagnosis of melioidosis. PLoS Negl Trop Dis 2014;8(3):e2727

129 Dance D. Treatment and prophylaxis of melioidosis. Int J Antimicrob Agents 2014;43(4):310-318

130 Lipsitz R, Garges S, Aurigemma R, et al. Workshop on treatment of and postexposure prophylaxis for Burkholderia pseudomallei and B. mallei Infection, 2010. Emerg Infect Dis 2012; 18(12):e2

131 Peacock SJ, Schweizer HP, Dance DA, et al. Management of accidental laboratory exposure to Burkholderia pseudomallei and B. mallei. Emerg Infect Dis 2008;14(7):e2

132 White NJ, Dance DA, Chaowagul W, Wattanagoon Y, Wuthiekanun V, Pitakwatchara N. Halving of mortality of severe melioidosis by ceftazidime. Lancet 1989;2(8665):697-701

133 Simpson AJ, Suputtamongkol Y, Smith MD, et al. Comparison of imipenem and ceftazidime as therapy for severe melioidosis. Clin Infect Dis 1999;29(2):381-387

134 Smith MD, Wuthiekanun V, Walsh AL, White NJ. Susceptibility of Pseudomonas pseudomallei to some newer beta-lactam antibiotics and antibiotic combinations using time-kill studies. J Antimicrob Chemother 1994;33(1):145-149

135 Smith MD, Wuthiekanun V, Walsh AL, White NJ. In-vitro activity of carbapenem antibiotics against beta-lactam susceptible and resistant strains of Burkholderia pseudomallei. J Antimicrob Chemother 1996;37(3):611-615

136 Cheng AC, Fisher DA, Anstey NM, Stephens DP, Jacups SP, Currie BJ. Outcomes of patients with melioidosis treated with meropenem. Antimicrob Agents Chemother 2004;48(5): 1763-1765

137 Huffam S, Jacups SP, Kittler P, Currie BJ. Out of hospital treatment of patients with melioidosis using ceftazidime in $24 \mathrm{~h}$ elastomeric infusors, via peripherally inserted central catheters. Trop Med Int Health 2004;9(6):715-717

138 Chierakul W, Anunnatsiri S, Short JM, et al. Two randomized controlled trials of ceftazidime alone versus ceftazidime in combination with trimethoprim-sulfamethoxazole for the treat- ment of severe melioidosis. Clin Infect Dis 2005;41(8): 1105-1113

139 Chierakul W, Anunnatsiri S, Chaowagul W, Peacock SJ, Chetchotisakd P, Day NP. Addition of trimethoprim-sulfamethoxazole to ceftazidime during parenteral treatment of melioidosis is not associated with a long-term outcome benefit. Clin Infect Dis 2007;45(4):521-523

140 Wuthiekanun V, Amornchai P, Saiprom N, et al. Survey of antimicrobial resistance in clinical Burkholderia pseudomallei isolates over two decades in Northeast Thailand. Antimicrob Agents Chemother 2011;55(11):5388-5391

141 Chantratita N, Rholl DA, Sim B, et al. Antimicrobial resistance to ceftazidime involving loss of penicillin-binding protein 3 in Burkholderia pseudomallei. Proc Natl Acad Sci U S A 2011; 108(41):17165-17170

142 Sarovich DS, Price EP, Von Schulze AT, et al. Characterization of ceftazidime resistance mechanisms in clinical isolates of Burkholderia pseudomallei from Australia. PLoS ONE 2012;7(2): e30789

143 Crowe A, McMahon N, Currie BJ, Baird RW. Current antimicrobial susceptibility of first-episode melioidosis Burkholderia pseudomallei isolates from the Northern Territory, Australia. Int J Antimicrob Agents 2014;44(2):160-162

144 Chetchotisakd P, Chierakul W, Chaowagul W, et al. Trimethoprim-sulfamethoxazole versus trimethoprim-sulfamethoxazole plus doxycycline as oral eradicative treatment for melioidosis (MERTH): a multicentre, double-blind, non-inferiority, randomised controlled trial. Lancet 2014;383(9919): 807-814

145 Dance DA, Davong V, Soeng S, Phetsouvanh R, Newton PN, Turner P. Trimethoprim/sulfamethoxazole resistance in Burkholderia pseudomallei. Int J Antimicrob Agents 2014;44(4):368-369

146 Jenney AW, Lum G, Fisher DA, Currie BJ. Antibiotic susceptibility of Burkholderia pseudomallei from tropical northern Australia and implications for therapy of melioidosis. Int J Antimicrob Agents 2001;17(2):109-113

147 Dance DA, Wuthiekanun V, Chaowagul W, Suputtamongkol Y, White NJ. Development of resistance to ceftazidime and coamoxiclav in Pseudomonas pseudomallei. [letter]J Antimicrob Chemother 1991;28(2):321-324

148 Dance DA, Wuthiekanun V, Chaowagul W, White NJ. The antimicrobial susceptibility of Pseudomonas pseudomallei. Emergence of resistance in vitro and during treatment. J Antimicrob Chemother 1989;24(3):295-309

149 Dance DA, Wuthiekanun V, White NJ, Chaowagul W. Antibiotic resistance in Pseudomonas pseudomallei. Lancet 1988;1(8592): 994-995

150 Chaowagul W, Simpson AJ, Suputtamongkol Y, Smith MD, Angus BJ, White NJ. A comparison of chloramphenicol, trimethoprimsulfamethoxazole, and doxycycline with doxycycline alone as maintenance therapy for melioidosis. Clin Infect Dis 1999;29(2): 375-380

151 Cheng AC, Chierakul W, Chaowagul W, et al. Consensus guidelines for dosing of amoxicillin-clavulanate in melioidosis. Am J Trop Med Hyg 2008;78(2):208-209

152 Cheng AC, McBryde ES, Wuthiekanun V, et al. Dosing regimens of cotrimoxazole (trimethoprim-sulfamethoxazole) for melioidosis. Antimicrob Agents Chemother 2009;53(10):4193-4199

153 Chaowagul W, Suputtamongkol Y, Dance DA, Rajchanuvong A, Pattara-arechachai J, White NJ. Relapse in melioidosis: incidence and risk factors. J Infect Dis 1993;168(5):1181-1185

154 Limmathurotsakul D, Chaowagul W, Chantratita N, et al. A simple scoring system to differentiate between relapse and re-infection in patients with recurrent melioidosis. PLoS Negl Trop Dis 2008; 2(10):e327

155 Lumbiganon P, Chotechuangnirun N, Kosalaraksa P, Teeratakulpisarn J. Localized melioidosis in children in Thailand: 
treatment and long-term outcome. J Trop Pediatr 2011;57(3): 185-191

156 Cheng AC, West TE, Limmathurotsakul D, Peacock SJ. Strategies to reduce mortality from bacterial sepsis in adults in developing countries. PLoS Med 2008;5(8):e175

157 Cheng AC, Stephens DP, Anstey NM, Currie BJ. Adjunctive granulocyte colony-stimulating factor for treatment of septic shock due to melioidosis. Clin Infect Dis 2004;38(1):32-37

158 Cheng AC, Limmathurotsakul D, Chierakul W, et al. A randomized controlled trial of granulocyte colony-stimulating factor for the treatment of severe sepsis due to melioidosis in Thailand. Clin Infect Dis 2007;45(3):308-314

159 Harris P, Engler C, Norton R. Comparative in vitro susceptibility of Burkholderia pseudomallei to doripenem, ertapenem, tigecycline and moxifloxacin. Int J Antimicrob Agents 2011;37(6):547-549
160 Estes DM, Dow SW, Schweizer HP, Torres AG. Present and future therapeutic strategies for melioidosis and glanders. Expert Rev Anti Infect Ther 2010;8(3):325-338

161 Sarkar-Tyson M, Atkins HS. Antimicrobials for bacterial bioterrorism agents. Future Microbiol 2011;6(6):667-676

162 Schweizer HP. When it comes to drug discovery not all Gramnegative bacterial biodefence pathogens are created equal: Burkholderia pseudomallei is different. Microb Biotechnol 2012; 5(5):581-583

163 Peacock SJ, Limmathurotsakul D, Lubell Y, et al. Melioidosis vaccines: a systematic review and appraisal of the potential to exploit biodefense vaccines for public health purposes. PLoS Negl Trop Dis 2012;6(1):1488

164 Choh LC, Ong GH, Vellasamy KM, et al. Burkholderia vaccines: are we moving forward? Front Cell Infect Microbiol 2013;3:5 